hexane was shaken with $2 \mathrm{ml}$. of boron trifluoride etherate, $\mathrm{BF}_{3}+\left(\mathrm{C}_{2} \mathrm{H}_{5}\right)_{2} \mathrm{O}$ (b.-p. $124-126^{\circ}$ ), at room temperature. The dark blue complex was decomposed by the addition of $100-200 \mathrm{ml}$. of $95 \%$ methanol, then transferred with water into hexane. The hypophase was discarded and the epiphase washed, dried, adsorbed on a suitable lime-celite column (2:1) and developed with hexane. The respective zones were eluted with acetone and transferred into hexane. The cis members of the stereoisomeric $\beta$-carotene set were identified as such by catalyzing with iodine and showing that the resulting all-trans pigment did not separate in the mixed chromatogram test from $\beta$-carotene ex carrots. Pertinent spectroscopic observations were also made. The hexane solutions of pigment zones I and II were completely evaporated in a dry nitrogen stream; the light orange colored powdery residues were dissolved in the minimum amount of benzene and crystallized at $50^{\circ}$ in a centrifuge tube by cautious addition of methanol $\left(50^{\circ}\right)$. The samples were recrystallized in the same manner. The curves given in the figures were taken in the Beckman instrument. The maxima, as observed visually in the Zeiss Evaluating Grating Spectroscope (LoeweSchumm) and compared with corresponding data for $\beta$-carotene, were located as follows: Pigment I: 448, $422 \mathrm{~m} \mu$ (in hexane); Pigment II : 468, $441 \mathrm{~m} \mu ; \beta$-Carotene: $484,454 \mathrm{~m} \mu$.

Summary. $-\beta$-Carotene when treated with boron trifluoride etherate for one minute yields mainly some of its cis forms; however, upon longer treatment several new pigments with shorter chromophores appear, two of which have been crystallized.

* Contribution No. 1806.

${ }^{1}$ Lewis, G. N., and Seaborg, G. T., J. Am. Chem. Soc., 61, 1886 (1939).

${ }^{2}$ Strain, H. H., Ibid., 63, 3448 (1941).

MENDELIAN AND NON-MENDELIAN FACTORS AFFECTING THE CYTOCHROME SYSTEM IN NEUROSPORA CRASSA*

By M. B. Mitchell, H. K. Mitchell, and A. Tissieres $\dagger$

Kerckhoff laboratories of Blology, Calipornia institute of Technology, Pasadena

Communicated by G. W. Beadle, May 4, 1953

It was reported recently that the characteristics of a slow-growing strain, called poky, are inherited maternally. ${ }^{1}$ This observation has focused attention on strains which grow slowly on complete medium and which have long been known to appear very frequently in material examined in order to detect nutritional mutants of Neurospora. An indication of the frequency of their appearance can be obtained from some figures taken from 
the data of Beadle and Tatum. ${ }^{2}$ Among 8795 ascospores isolated from irradiated material during their study by one of the present authors ( $M$. B. M.), 7049 germinated. Of these, 54 gave rise to strains having easily diagnosed requirements for components present in the complete medium used and, therefore, represented the class of nutritional mutants sought. A much greater number, 489 , were distinguishable from wild type because of their slower growth on complete medium. Of these 159 did not grow sufficiently to allow their being tested. The remaining 330 were discarded, either because they grew too rapidly on minimal medium, or because they did not give a well-defined response to the growth-factor mixtures present in the complete medium. Similar figures have been reported by Lein, Mitchell, and Houlahan ${ }^{3}$ in a description of a variation in the method of Beadle and Tatum. ${ }^{2}$

The advent of poky has not only aroused curiosity as to the nature of the abnormalities associated with the slow growth of such strains but has also suggested ways in which an investigation of them might be started, namely, by learning the mode of inheritance and by examining the cytochrome system for abnormalities such as those exhibited by poky.4, 5 Among slow-growing strains recently isolated and studied, three have been found which show abnormalities with respect to cytochromes. The differences from normal are inherited maternally in one of these strains and as gene mutations in the other two. The four strains, poky and the three to be described here, are all different phenotypically. Ephrussi and his collaborators $^{6}$ have described, in yeast, a segregational mutant and a vegetative mutant both of which are characterized by the same disturbances of the cytochrome and succinic oxidase systems. A strain of yeast described by Ycas and Starr $^{7}$ has, in addition to these disturbances, other abnormalities which are inherited as if due to gene mutation.

Inheritance.-The three Neurospora strains were found among single ascospore isolates from a cross of wild type Abbott 12a protoperithecia to wild 1400-4A conidia, both untreated. The spores were plated on minimal medium and examined after about 15 hours of incubation at $25^{\circ} \mathrm{C}$. when slow-growing ones were isolated. Strains from six isolates showed maternal inheritance of the abnormalities but since they have not been shown to be different, only one, designated mi-3, will be considered here. (The designation abbreviates "maternally inherited," thus poky is mi-1.) Strains showing Mendelian inheritance of the mutant trait included one arginine mutant and the two nuclear gene mutants described here, designated $\mathrm{C} 115$ and $\mathrm{C} 117$.

The mi-3 character descends to all progeny of crosses of mi-3 protoperithecia to normal conidia and to none of the progeny of reciprocal crosses, normal protoperithecia to mi-3 conidia. (In designating crosses, the protoperithecial parent will be given first.) Asci were isolated from mi-3 $\times$ 
wild 1400-4A and the strains from the four spore pairs of one ascus (number 2010) were crossed to wild types 2293-2a and 2292-2A. Spores were plated on minimal medium, heat treated, incubated at $25^{\circ} \mathrm{C}$. for 12 to 20 hours and counted as follows:

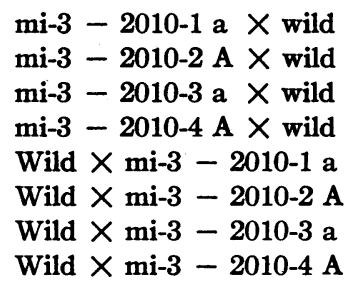

$\begin{array}{rc}\text { WIID } & \text { sLow } \\ 2 & 962 \\ 0 & 473 \\ \ldots & \ldots \text { (not fertile) } \\ 0 & 1368 \\ 1044 & 9 \\ 1154 & 5 \\ 1113 & 3 \\ 1059 & 7\end{array}$

Crosses of strains derived from an ascus (number 2543) from the cross mi-3 - 2010 - 4A $\times$ wild 2293-2a gave similar results, which appear below.

mi-3 $-2543-1$ a $\times$ wild
mi-3 $-2543-2$ A $\times$ wild
mi-3 $-2543-3$ a $\times$ wild
mi-3 $\times 2543-4$ A $\times$ wild
Wild $\times$ mi-3 $-2543-1$ a
Wild $\times$ mi-3 $-2543-2$ A
Wild $\times$ mi-3 $-2543-3$ a
Wild $\times$ mi-3 $-2543-4$ A

$\begin{array}{rr}\text { WIID } & \text { sLow } \\ 0 & 902 \\ 0 & 1269 \\ 0 & 1327 \\ 0 & 2071 \\ 1067 & 8 \\ 842 & 7 \\ 1103 & 15 \\ 1174 & 13\end{array}$

Very few spores which had failed to germinate were observed. Occasional wild offspring from mi- $3 \times$ wild crosses are attributed, as in the case of poky, ${ }^{1}$ to the formation of a few wild protoperithecia derived from the fertilizing conidia. Slow progeny from wild $\times$ mi-3 crosses are thought to be due to late germination or to factors which cause slow growth but are independent of mi-3 since those which were isolated and tested did not behave like mi-3. Crosses of mi-3 $\times$ mi-3 gave only slow progeny among 11,318 spores plated as above.

Because of the similarities between poky and mi-3 it appeared possible that one is a modified form of the other. In order to learn whether a segregating modifier is involved reciprocal crosses were made between po1720-1a and mi-3-2010-1A. Strains derived from the four spore pairs of each of five asci from each cross were cultured at $25^{\circ} \mathrm{C}$. in liquid minimal medium in $125-\mathrm{ml}$. flasks. The lowest, highest, and average dry weights in $\mathrm{mg}$. for each set of 20 six-day cultures are given below.

$\begin{array}{lcc} & \text { poky } \times \text { mi-3 } & \text { mi-3 } \times \text { poky } \\ \text { Lowest } & 2 & 36 \\ \text { Highest } & 24 & 59 \\ \text { Average } & 13 & 49\end{array}$

Dry weights from poky $\times$ mi-3 progeny are typical of those from poky $\times$ wild progeny and dry weights from mi-3 $\times$ poky progeny are likewise typical of those given by progeny of mi-3 $\times$ wild. From these results it ap- 
pears that if a modifier accounts for the difference in growth rates of poky and mi-3, the modifier is itself inherited maternally. Hence, it may be supposed that poky and mi-3 differ from each other, as well as from wild type, by a maternally inherited trait.

Mutants C115 and C117 were crossed, as conidial parents, to wild and to several other mutants. Counts of spores plated on minimal medium are given below.

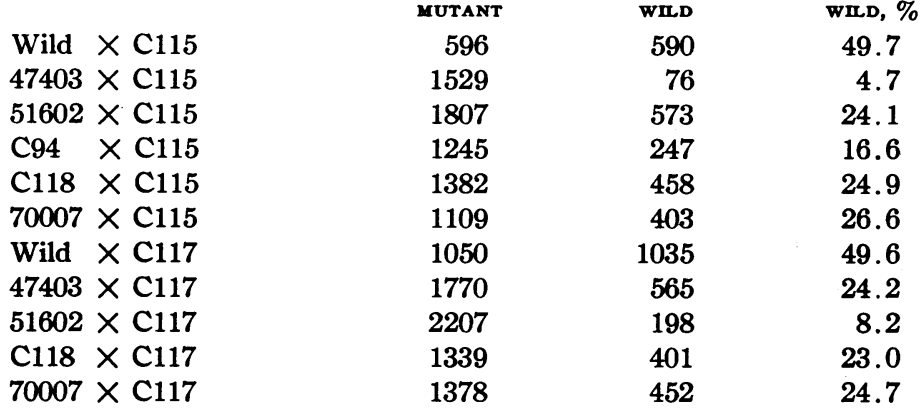

The other mutants involved in these crosses are as follows:

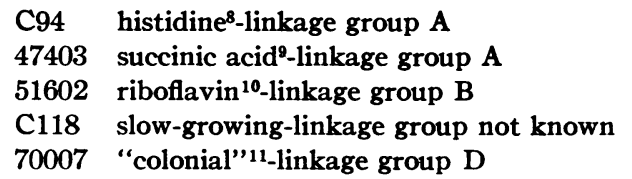

In 59 asci from crosses of $\mathrm{C} 115$ and in 127 from crosses of $\mathrm{C} 117$ there was $1: 1$ segregation of the mutant gene and its wild allele. These data indicate that the slow-growth character is due in each case to gene mutation; and that the $\mathrm{C} 115$ locus is in linkage group $\mathrm{A}$ and the $\mathrm{C} 117$ locus in linkage group B.

$\mathrm{C} 115$ and $\mathrm{C} 117$ have not been observed to function as protoperithecial parents. Both strains form protoperithecia but when these are fertilized they either stop developing after enlarging slightly and turning brown or they do not appear to change at all. Occasionally a few mature perithecia appeared belatedly but these could have arisen from protoperithecia formed by the fertilizing strain. Those which have been examined, from crosses to wild, showed 1:1 segregation of mutant and wild. All crosses attempted between $\mathrm{C} 115$ and $\mathrm{C} 117$ isolates were completely sterile. It seems probable, however, since the two mutants show linkage to different groups, that they are genetically different.

Preparation of Recombinants.- $\mathrm{C} 115$ in poky and mi-3 and $\mathrm{C} 117$ in mi-3 were obtained from asci from crosses to poky and mi-3 protoperithecia. The presence of poky or mi-3 in the recombinant strains seems unquestionable since from each ascus examined, strains from the two spore pairs not carrying the mutant genes were typically poky or mi-3. The presence of the 
mutant genes was demonstrated by the observation of $1: 1$ segregation of mutant and wild in asci and among random spores from crosses of the recombinants to wild-type protoperithecia. Counts of random spores germinated on minimal plates are as follows:

$\begin{array}{lccc} & \text { WILD } & \text { MUTANT } & \text { MUTANT, \% } \\ \text { Wild } \times \text { C115 in poky } & 835 & 857 & 50.6 \\ \text { Wild } \times \text { C115 in mi-3 } & 759 & 752 & 49.8 \\ \text { Wild } \times \text { C117 in mi-3 } & 861 & 902 & 51.2\end{array}$

Several mutant isolates from asci from each of these crosses were tested and proved to have the same characteristics as the mutant parent in the cross to poky or mi-3.

So far no isolate of $\mathrm{C} 117$ in poky has grown enough to permit its being thoroughly tested and out-crossed. Segregation of the mutant gene could be observed among spore pairs from 24 asci from poky $\times \mathrm{C} 117$ isolated and allowed to germinate on plates of minimal medium. Two spore pairs from each ascus had produced, after about 20 hours, the amount of growth typical of poky and, when transferred to agar slants, continued to grow and formed typical poky cultures. The other two pairs in each ascus had, after 20 hours, produced hyphae not much longer than the ascospore and, when transferred to slants, these hyphae, in most cases, grew very little more and finally disintegrated. In a few cultures growth continued very slowly but after three weeks the agar surface in 3-inch test-tube slants was not covered with mycelium. Two such isolates have been kept alive by transferring to fresh slants bits of agar containing mycelium. Recently it has been found that their growth rate can be increased slightly by varying the complete medium used.

Growth Characteristics and Differences in Content of Cytochromes.-The following are typical dry weights of mycelium obtained from four-day cultures of the various strains at $25^{\circ} \mathrm{C}$. in 125 -ml. flasks containing $20 \mathrm{ml}$. of minimal medium with and without a supplement consisting of $40 \mathrm{mg}$. per flask of Difco yeast extract. A number of isolates of each strain (with the exception of $\mathrm{C} 117$ in poky) have been tested.

Dry Weights, Mg.

$\quad$ straIn
Wild type
poky
mi-3
C115
C117
C115 in poky
C117 in poky
C115 in mi-3
C117 in mi-3

\begin{tabular}{rl}
\multicolumn{1}{c}{ Minimal } & \multicolumn{2}{c}{ Yeast extract } \\
75 & 95 \\
11 & 14 \\
40 & 53 \\
30 & 2 \\
24 & 26 \\
4 & $<0.5$ \\
$<0.5$ (6 days) & $14 \quad$ (6 days) \\
6 & $<0.5$ \\
23 & 24
\end{tabular}


In older cultures in flasks and on agar slants, a difference in the growth rates of $\mathrm{C} 115$ in poky and $\mathrm{C} 115$ in mi-3 becomes apparent, the latter strain being faster. The inhibition of $\mathrm{C} 115$ by yeast extract has been characteristic of all isolates examined but no inhibitory substance has been identified. Flask cultures of $\mathrm{C} 117$ in poky were inoculated with small bits of agar containing mycelium since the isolates have so far produced very few aerial hyphae and conidia.

The differences in intensity of absorption bands due to cytochromes are indicated in figure 1. The observations were made at room temperature with a hand spectroscope on moist pads of mycelium grown for four days at

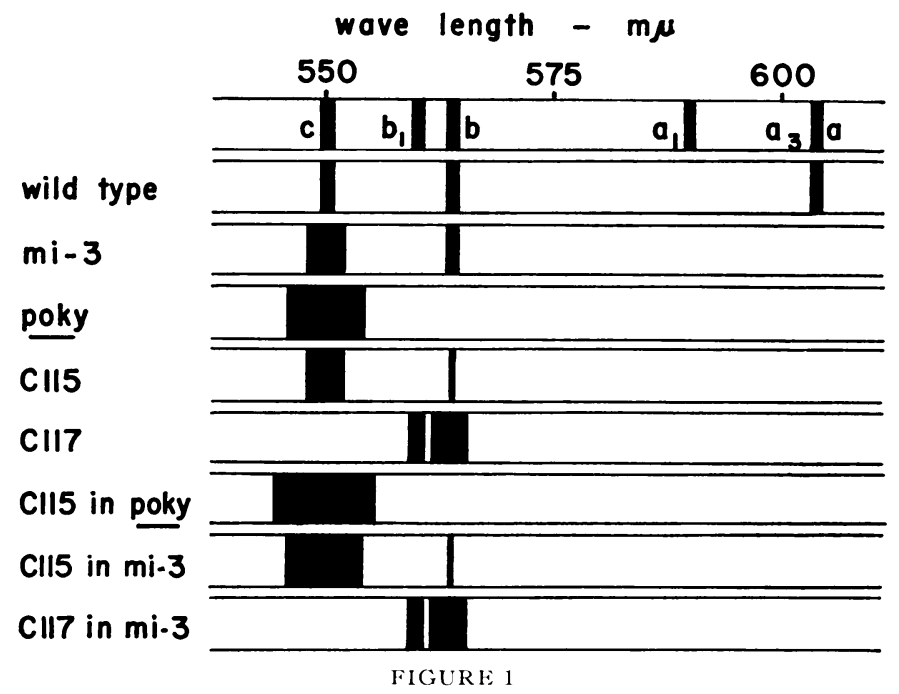

Diagrammatic representation of cytochrome absorption bands. Relative concentrations of cytochromes are indicated by the width of the bands, e.g., the ratio of cytochrome $c$ concentration in wild type, mi-3 and poky is approximately $1: 5: 15$.

$2.5^{\circ} \mathrm{C}$. on minimal medium in $12.5-\mathrm{ml}$. flasks. The pads were first treated with sodium hydrosulfite. Several cultures of the slower strains were used in order to obtain pads weighing about $30 \mathrm{mg}$. dry. It will be seen that poky, mi-3, and C115) are rather similar but the differences indicated, between cultures of this age, have been consistent. The $b$ band is normal in mi-3, weaker than normal in C 115 , and very weak or not visible in poky. The $c$ band is stronger in poky. The changes in poky which occur with increasing age have been described. ${ }^{+}$Briefly, older mycelium is more like that of wild type. This may be true to some extent, also, of mi-3 but not of $\mathrm{C} 115$ and $\mathrm{C} 117$. In $\mathrm{C} 117$ the $c$ band has never been observed; the $b$ band has been consistently stronger than normal; and the band which may cor- 
respond to that of cytochrome $b_{1}$, not so far observed in any strain not carrying $\mathrm{C} 117$, has always been present. The absorption band of cytochrome $a$ has not been observed in mycelium of this age from any of the strains. A band which may correspond to cytochrome $a_{1}$ is sometimes observed but this has not been consistent with any strain or even with any one isolate. It is usually seen in mycelium from mi-3, however.

With respect to content of cytochromes the characteristics of the recombinants are much as might be predicted from the properties of the individual strains. Cytochrome $c$ is accumulated by poky, mi-3 and $\mathrm{C} 115$ and both $\mathrm{C} 115$ in poky and $\mathrm{C} 115$ in mi-3 show an increased accumulation, the greater increase in $\mathrm{C} 115$ in poky corresponding to the greater excess in poky. Since cytochrome $b$ is normal in mi-3, weak in C115 and not detectable, by the method used, in poky, it is not surprising to find that this band is weak in $\mathrm{C} 115$ in mi-3 and not detectable in $\mathrm{C} 115$ in poky. That $\mathrm{C} 117$ in mi-3 does not differ from $\mathrm{C} 117$ is not surprising if, as it appears, $\mathrm{C} 117$ prevents the appearance of cytochrome $c$ and induces an increase in cytochrome $b$ in cytoplasm which, in the absence of the mutant gene, is normal with respect to $b$. On the basis of the characteristics of poky and $\mathrm{C} 117$ it might be predicted that only cytochrome $b_{1}$ could be detected in $\mathrm{C} 117$ in poky. In the few pads which have been examined the $a$ and $c$ bands could not be detected but a very weak and diffuse band in the region of $b$ and $b_{1}$ could be seen. The band which may correspond to that of $a_{1}$ could be seen also, but it was weak. The behavior of the recombinants appears to emphasize the differences shown by poky, mi-3 and C115. Other differences have been demonstrated by comparisons of enzyme activities, to be reported elsewhere.

Mixed Cultures.-Mixed cultures have been studied in an attempt to learn whether these strains can interact to produce normal mold, but in no case has satisfactory evidence of such interaction been obtained. The mixture of poky and mi-3 was examined in particular since it appeared to offer more interesting possibilities. Different isolates, different conditions, and different means of testing the products (cultures established from hyphal tips and single conidia from the mixture and single ascospores from crosses in which the mixture was protoperithecial parent) were tried but no mold with a growth rate significantly higher than that of mi-3 was detected. It seems possible that the growth rate of a strain arising from a mixture of poky and mi-3 cytoplasms might be intermediate between that of poky and mi-3. Such a strain would be difficult to detect in the mixture.

Summary.-Three strains of Neurospora, obtained from a cross of untreated standard wild types, show differences from wild type in growth rate and in cytochrome content which are similar to those exhibited by the poky strain, previously described. In one of these the character, designated mi-3, is, like poky, inherited maternally and the differences between poky and mi-3 appear to be inherited maternally. The other two characters, 
designated $\mathrm{C} 115$ and $\mathrm{C} 117$, are inherited as gene differences. $\mathrm{C} 115$ and mi-3 both have an excess of cytochrome $c$ but not as great as in poky and both, like poky, are deficient in cytochrome $a$. The content of cytochrome $b$ is normal in mi-3 and low in $\mathrm{C} 115$ but not as low as in poky. Cytochromes $a$ and $c$ have not been detected in $\mathrm{C} 117$ but there is an excess of cytochrome $b$. The three strains grow more slowly than wild but faster than poky. Strains which carry the mutant genes along with the maternally inherited characters have also been examined. Their properties are much as would be predicted from the characteristics of the individual components.

* This work was supported in part by funds from the Rockefeller Foundation and by funds from the Atomic Energy Commission administered through contract with the Office of Naval Research, U. S. Navy. Contract N6-onr 244. Task Order V.

$\dagger$ Gosney Fellow. Present address: Molteno Institute, Cambridge University, Cambridge, England.

1 Mitchell, M. B., and Mitchell, H. K., Proc. Natl. Acad. Scr., 38, 442 (1952).

2 Beadle, G. W., and Tatum, E. L., Am. J. Bot., 32, 678 (1945).

${ }^{3}$ Lein, J., Mitchell, H. K., and Houlahan, M. B., Proc. Natl. Acad. Sci., 34, 435 (1948).

4 Haskins, F. A., Tissieres, A., Mitchell, H. K., and Mitchell, M. B., J. Biol. Chem., 200, 819 (1953).

5 Tissieres, A., Mitchell, H. K., and Haskins, F. A. (in press).

- Ephrussi, B., and Hottinguer, H., Cold Spring Harbor Symposia on Quantitive Biology, XVI, 75 (1951).

7 Ycas, M., and Starr, T. J., J. Bact., 65, 83 (1953).

${ }^{8}$ Haas, F., Mitchell, M. B., Ames, B. N., and Mitchell, H. K., Genetics, 37, 217 (1951).

9 Fincham, J. R. S., J. Biol. Chem., 182, 61 (1950).

${ }^{10}$ Houlahan, M. B., Beadle, G. W., and Calhoun, H. G., Genetics, 34, 493 (1949).

1 Mitchell, M. B., Pittenger, T. H., and Mitchell, H. K., Proc. Nart. Acad. Scr., 38, 570 (1952).

\section{INDUCTION OF CHROMOSOME BREAKAGE AT MEIOSIS BY A MAGNESIUM DEFICIENCY IN TRADESCANTIA}

\section{By Dale Steffensen}

\section{Department of Genetics, University of California, Bei keley*}

Communicated by R. E. Clausen, April 24, 1953

The mineral elements as constituents of the nucleus undoubtedly participate in important structural and metabolic functions of the nuclear components. Microincineration studies of $\operatorname{Scott}^{1,3}$ and Barigozzi ${ }^{2}$ showed nuclear minerals, mostly calcium and magnesium, to be closely associated with the chromosomes. Allgén ${ }^{4}$ found magnesium to be a constituent of nucleo-histone. Gulick ${ }^{5}$ and, more recently, Milovidov 6 have reviewed the problem of inorganic elements within the nucleus. Poulson and Bowen ${ }^{7}$ have used radioactive tracer methods in further explorations in this field. 